\title{
APUNTES SOBRE LAS CRUCES DE PLATA DE YALALAG
}

\author{
POR
}

NORMAN P. WRIGH T

TE TENIDo ocasión de estudiar algunas cruces de plata talladas en la. 11 Sierra de Juárez, Oaxaca, y en la inteligencia de que apenas han sido estudiados estos legados de la época colonial, me permito someter los apuntes siguientes, los cuales, aunque carezcan de la documentación necesaria para un estydio comprensivo, suman por lo menos los hechos que he podido constat. en la materia y agregan unas hipótesis y algunas preguntas que necesitan aclaración para explicar satisfactoriamente el origen de estos objetos.

Este modesto trabajo es pues, solamente, un principio; para completarlo se hecesitan todos los datos que puedan conseguirse $y$, sobre todo, cualquier informe que se pueda sacar de los archivos coloniales del Estado fe Oaxaca o de los anales de las órdenes religiosas de ese Estado.

Las autoridades que hasta ahora he podido consultar sobre dichos archivos poco han contribuido; no obstante, ¿ seria demasiado optimismo suponer que escondido en las escrituras del pasado, haya algo que nos 
ayude a esclarecer en qué lugar, y por qué los indios de la región, únicos en México - según creo-, las adoptaron y las han lievado puestas hasta hoy día? No es posible que en otras partes de México se hayan fabricado cruces semejantes, en la época colonial, pero después de numerosos viajes a diversas y remotas regiones de la República, puedo decir que nunca he visto ningún vestigio de ellas. 1

Conocí al principio una pequeña colección de estas cruces en la ciudad de Oaxaca y me enteré de que fueron encontradas entre las familias acomodadas de Yalalag (Villa Hidalgo), en la cabecera de Villa Alta. La castualidad me llevó a esta remota ciudad zapoteca en noviembre del año de 1944 y acordándome de las graciosas cruces que habia visto, busqué informes y puede confirmar que aún las poseen las más ricas familias yalaltecas y que no es cosa rara que las mujeres las lleven puestas, suspendidas de rosarios, alrededor del cuello o encima del fino huipil que visten estas indias de aspecto tan digno y encantador. Las mujeres con quienes hablé sobre este asunto, ignoraban de dónde habían venido las cruces, pero las consideraban "muy viejas", diciendo que habian pertenecido a sus familias a través de generaciones. En el idioma zapoteca se habla de estas cruces como "Cruz yon", eso es, "Cruz antigua".

Después pude examinar otros ejemplares —en total treinta y ochoque se dividen en dos tipos básicos. Fuera de las que existen en Yalalag, y por supuesto, en otros pueblos de la comarca, he visto en total alrededor de setenta ejemplares, la mayoria en posesión de coleccionistas o personas aficionadas al arte. De vez en cuando se ve una u otra en las tiendas de antigüedades en la capital o en Puebla.

Entre las cruces vistas en Yalalag había también algunos ejemplares de fabricación moderna, muy inferiores a los tipos antiguos y mostrando influencia ajena o bien comercial. Traté de localizar en la ciudad un taller y otro posible centro de origen, pero en vano, nadie pudo decirme donde fueron fabricadas estas nuevas cruces. Después of hablar de un taller donde todavía se entallan cruces en madera, que muestran las influencias de las formas antiguas de plata. ¿No indican estos hechos que, desde el principio, no Yalalag, sino otro lugar en la comarca, ha sido el centro de fa. bricación, y que los yalaitecas, por ser la gente "bien" en la región, consiguieron la mayoría de ellas, $y$ adoptaron la costumbre de llevarlas?

1 En Cobán, Guatemala, vi dos cruces de lámina de plata, llevando tres sagrados corazones colgados. No tuve oportunidad de imvestigar ni su origen, ni la época en que fueron hechas. 
Una fuente autorizada me dice que también existen cruces semejantes talladas en oro, pero no las puedo describir por no haber visto ninguna de ellas.

Como queda dicho, las cruces viejas que yo personalmente he podido examinar se clasifican en dos variedades: el primer tipo, del cual he examinado seis, consiste en una lámina cruciforme sencilla, más o menos delgada, ostentando grabados en sus dos lados los emblemas de la Pasión. Las cuatro extremidades llevan un motivo que podría ser una estilización de tres plumas o de un cascabel, semejante a las extremidades de las cruces de piedra que se encuentran en los atrios de algunas de las iglesias más antiguas del pais. La técnica tosca de la grabación indica con bastante claridad el origen indio del grabador $y$ es también comparable en ciertos sentidos con la técnica en el detalle de esculturas del llamado "plateresco indio" de las iglesias coloniales.

De los seis ejemplares, cuatro son parecidos, pero no idénticos, pues tienen el detalle del dibujo distinto en cada caso. Sus medidas son: altura, 78 mms.; anchura, 45 mms.; anchura de los brazos, 8 mms. E1 quinto ejemplar es más grande y más macizo, con una altura de $90 \mathrm{mms}$., una anchura de 58 mms., y una anchura de los brazos de $10 \mathrm{mms}$. (foto núm. 3). La sexta cruz es distinta, siendo más delgada, y mide $105 \mathrm{mms}$. de altura por 77 mms. de anchura. Además, sus extremidades están algo apuntadas en lugar de mostrar el motivo indicado arriba.

Como se ve en las ilustraciones, este tipo de cruz carece de anillos, y está provisto de un agujero sencillo en la parte superior para suspenderla del rosario o collar.

La otra clase de cruz es más elaborada y muestra el empleo de un molde. Según mis observaciones, existe en mayor cantidad y tiene muchas variedades, no obstante el uso del molde. Sus lineas vigorosas y sus cuerpos redondeados y amplios ofrecen un contraste fuerte $y$ sorprendente con el aspecto angular $y$ austero de las otras. Parece que aquí tenemos un objeto europeb, por decirlo asi. Las pruebas de trabajo indio no se ven, aunque se puede suponer que fueron indios puros los que las hicieron.

De cada brazo y de la extremidad inferior de estas cruces cuelgan otras pequeñas, con menos frecuencia medallas o ambas cosas. La estructura de la crux principal varía entre una forma muy ornamentada $y$ algo más sencillo. I a mayor que he medido, en treinta y dos, tiene una altura máxima (incluyendo el anillo superior) de $185 \mathrm{mms}$ y una anchura máxima de 65 mms. (foto núm. 4). La menor de la serie mide $73 \mathrm{mms}$, por 32 mms. (foto núm. 5 ). 
De lás muchas formas diversas, la más común tiene en el centro una abertura en forma de corazón, flanqueada por alas, $y$ dos o tres ejemplares tienen aberturas redondas con pedazos de cristal (o piedra translúcida) azul o roja.

Otra versión que se repite es aquella que ostenta un cuadrado sencillo en el centro, colocado diagonalmente. $\mathrm{Da}$ la impresión de ser el tipo más primitivo de las formas moldeadas. Según un informe, este tipo se hacia antes en Santiago Comaltepec, pueblo no lejos de Choapan, un poco al este de Yalalag. Esto dijeron en Yalalag a un amigo fío que también se interesaba en las cruces. Si se pudiera confirmar esto, ¿no sería posible encontrar allí el punto de origen de todos los tipos, y que Yalalag se asocie con él por ser un lugar más importante, hasta donde penetran de vez en cuando los forasteros del mundo exterior?

El doctor Hildburgh, autoridad inglesa en el folklore espaniol, no conoce ningún tipo de cruz española que lleve cruces subordinadas colgadas de sus brazos, como los ejemplares moldeados que estudiamos. ${ }^{1}$

Este tipo, pues, parece no tener precedente. Este hecho sugiere una pregunta interesantísima: ¿ por qué nació en la lejana sierra de Juárez, región completamente india, una popularidad entre los indigenas para un objeto tan español como una cruz, y una cruz que tiene una nueva característica, sin precedente en España, aunque de índole cristiana?

Un punto suplementario que necesita aclaración es la identidad de los que originalmente poseian las cruces. EI número que se supone en existencia indica una distribución considerable. Si en efecto fueron cruces de rosario, ¿ puede ser que todo indio adulto llevara en esos días, como símbolo de cristianismo, an rosario provisto con una cruz tan preciosa y complicada?

Los tipos que tienen una, dos o tres medallas, en lugar de cruces subordinadas, facilitan la primera información concreta sobre todas estas cruces, por la lectura de sus inscripciones. Las medallas que he podido examinar llevan referencia a San Elías o a San Jorge.

San Elías es nuestra primera clave, por ser un santo venerado por los carmelitas. Una inscripción que he podido descrifrar es "S. Elías $P$.

1 Entiendo que la cruz más semejante de que tiene conocimiento el doctor Hildburgh es una forma relacionada con las llamadas "cruces de Caravaca", enpleadas como amuletos contra la brujería. Esta forma lleva a veces crecientes colgadas de los brazos de la cruz principal. Parece difícil establecer un enlace entre esta forma y la cruz moldeada de Yalalag. El motivo de los emblemas de la Pasión como los lleva la cruz de lámina, sí tiene precedente en España. 
Pop. D. Carm.", y una cruz que lleva esta leyenda fué reconocida como de origen carmelita en un convento de esta Orden en Londres. "P. Pop." podría ser "Pater Populi"; "D. Carm.", sin duda alguna se refiere a la Orden de los Carmelitas Descalzos.

El retrato de San Jorge, a veces al revés de la misma medalla, lleva la inscripción "S. Giorgius O. P." Aunque las iniciales "O, P." representan la Orden de los Dominicanos, esta orden nunca retrataba a San Jorge especialmente, $y$ un fraile dominicano, en Londres, no pudo reconocer la medalla en cuestión como un producto de su Orden.

Tampoco el motivo del Corazón Sagrado, mencionado arriba, constituye prueba de influencia jesuita, aunque no seria imposible interpretarlo así.

En estas condiciones, parece que la Orden de los Carmelitas, probablemente, inspiró algunas de estas cruces, si no todas ellas.

En cuanto a la edad de las distintas formas, mientras en México se suponía que los ejemplares de lámina grabada eran más antiguos (¿principios del siglo xvir?) que los tipos moldeados (¿ fines del siglo xvi y principios del xvir!?), los expertos del Museo Victoria and Albert de Londres, comparando su técncia de ejeeución con normas europeas, creen que ciertas de las cruces moldeadas (por ejemplo, la mayor) son las más antiguas. Ia cruz grande sería de principios del siglo xvmr, aunque manifiesta indicaciones de la técnica del siglo anterior. Todas las demás fornas serían del siglo xvin también, aunque algo más tarde. Del típo indicado como de origen de Santiago Comaltepec, se opina que es algo decadente, y de las cruces de lámina se cree que posiblemente fueron talladas en otro lugar, independientemente de las demás, y que un fraile pudo haber prestado un prototipo español a los indigenas para que lo copiaran.

Por la ausencia de datos referentes a estas cruces en los archivos del Estado, sugiero, to primer lugar, que se investigue en qué pueblos de la región la Orden Carmelita estableció sus misiones, sobre todo misiones dedicadas a Sart Elías o a San Jorge. ¿Hubo antes, por ejemplo, una misión carmeliti en Santiago Comaltepec? En Londres no he podido conseguir ningún.informe sobre este particular, fuera del hecho que la Orden estableció en el pais un total de veinte misiones de frailes y diez de monjas.

La presencia de tipos modernos en Yalalag indica que en algún lugar de estas remotas sierras no ha muerto la tradición de fabricar estos objetos. En este caso, si fuera posible hacer una serie completa de las formas predominantes a través de los siglos, contribuiria en gran escala a llenar las muchas lagunas que estoy obligado a dejar en este artículo. 


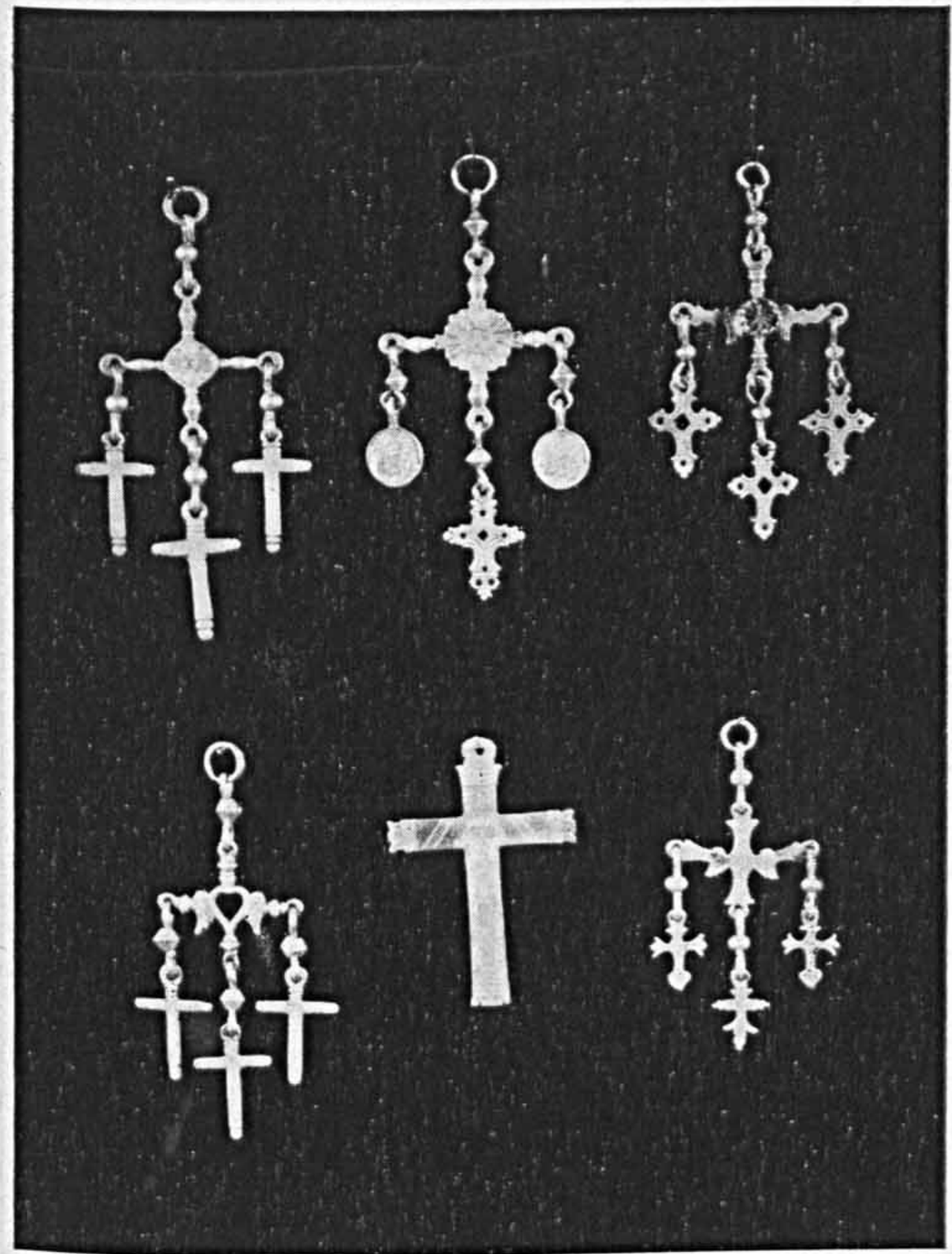

1. Colección de cruces de Yalalag. Tamaño reducido. En la parte inferior izquierda un corazón con alas 


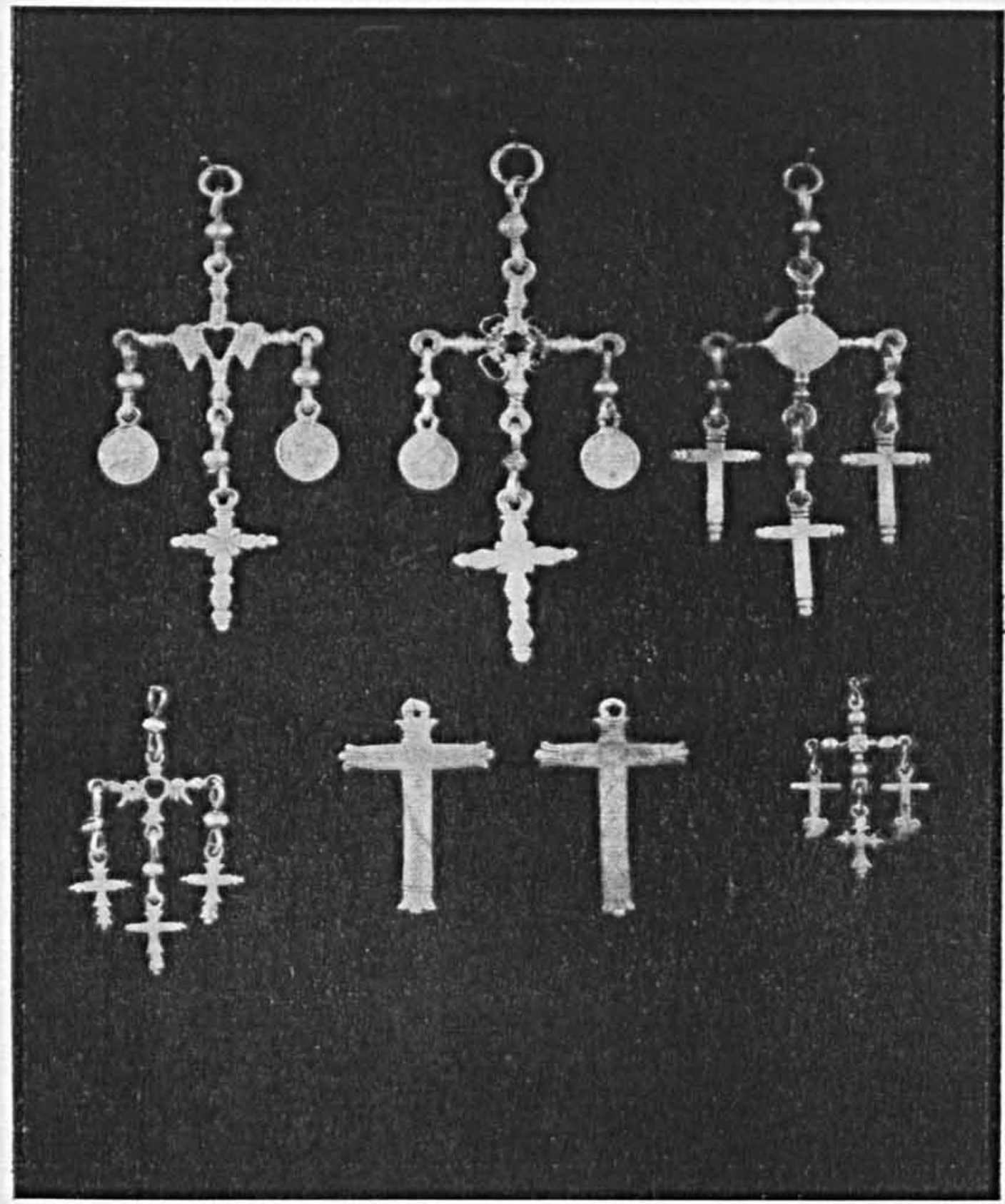

2. Colección de cruces de Yalalag. Tamaño reducido. Parte superior derecha el tipo señalado como de origen de Santiago Comaltepec 
DOI: http://dx.doi.org/10.22201/iie.18703062e.1948.16.458

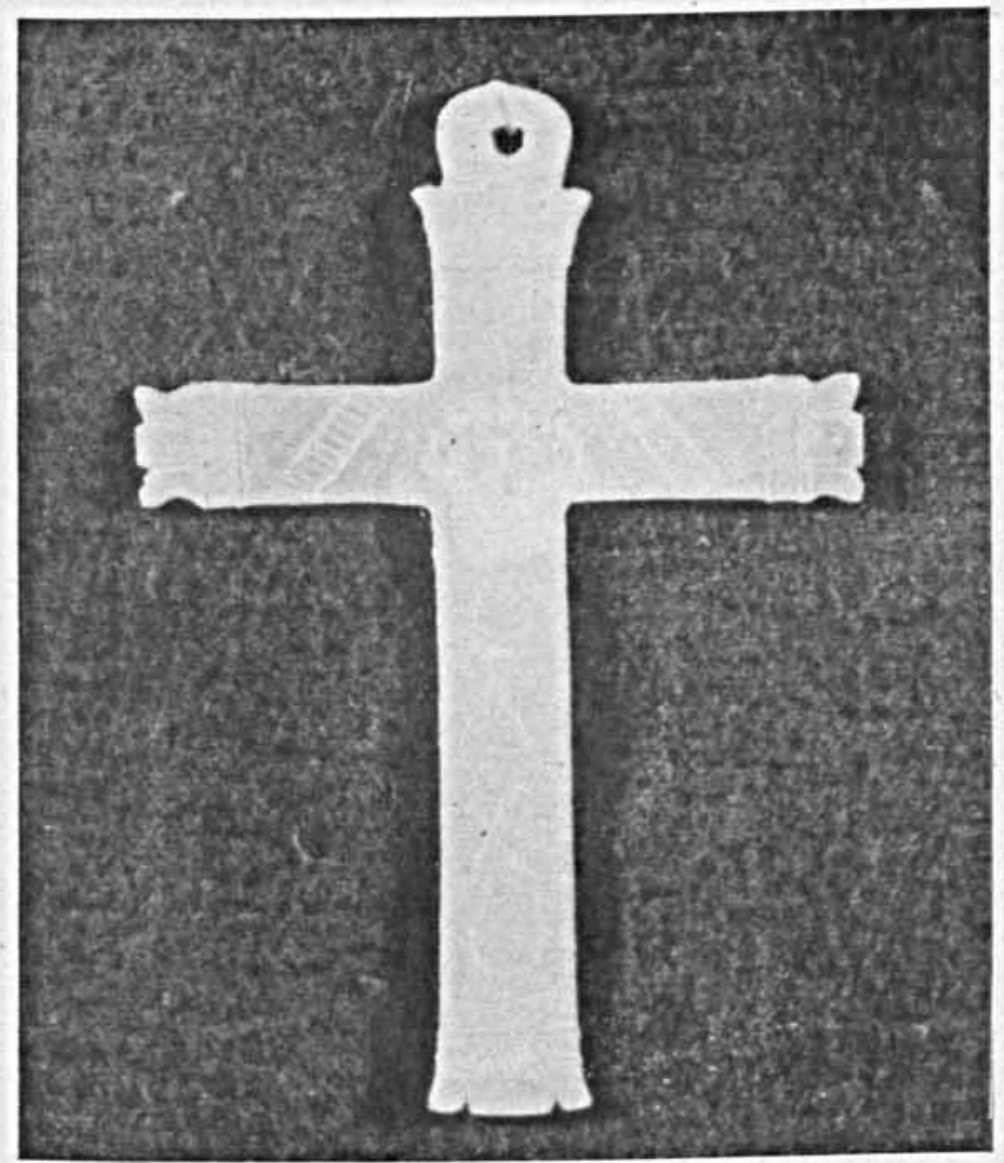

3. Cruz de lámina de plata con los emblemas de la Pasión. Tamaño natural 
DOI: http://dx.doi.org/10.22201/iie.18703062e.1948.16.458

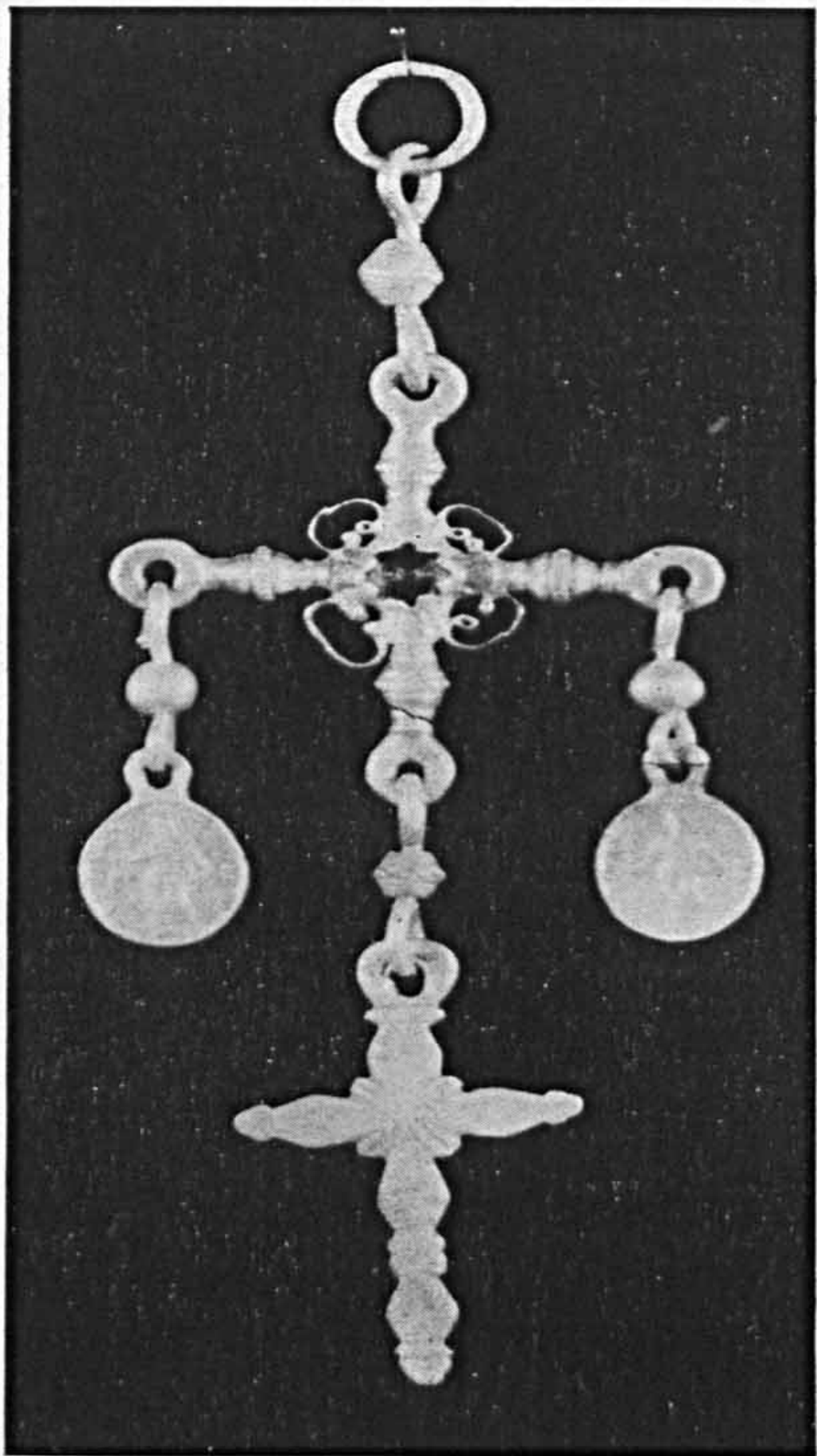

4. Cruz con las medallas de San Elias y San Jorge 
DOI: http://dx.doi.org/10.22201/iie.18703062e.1948.16.458

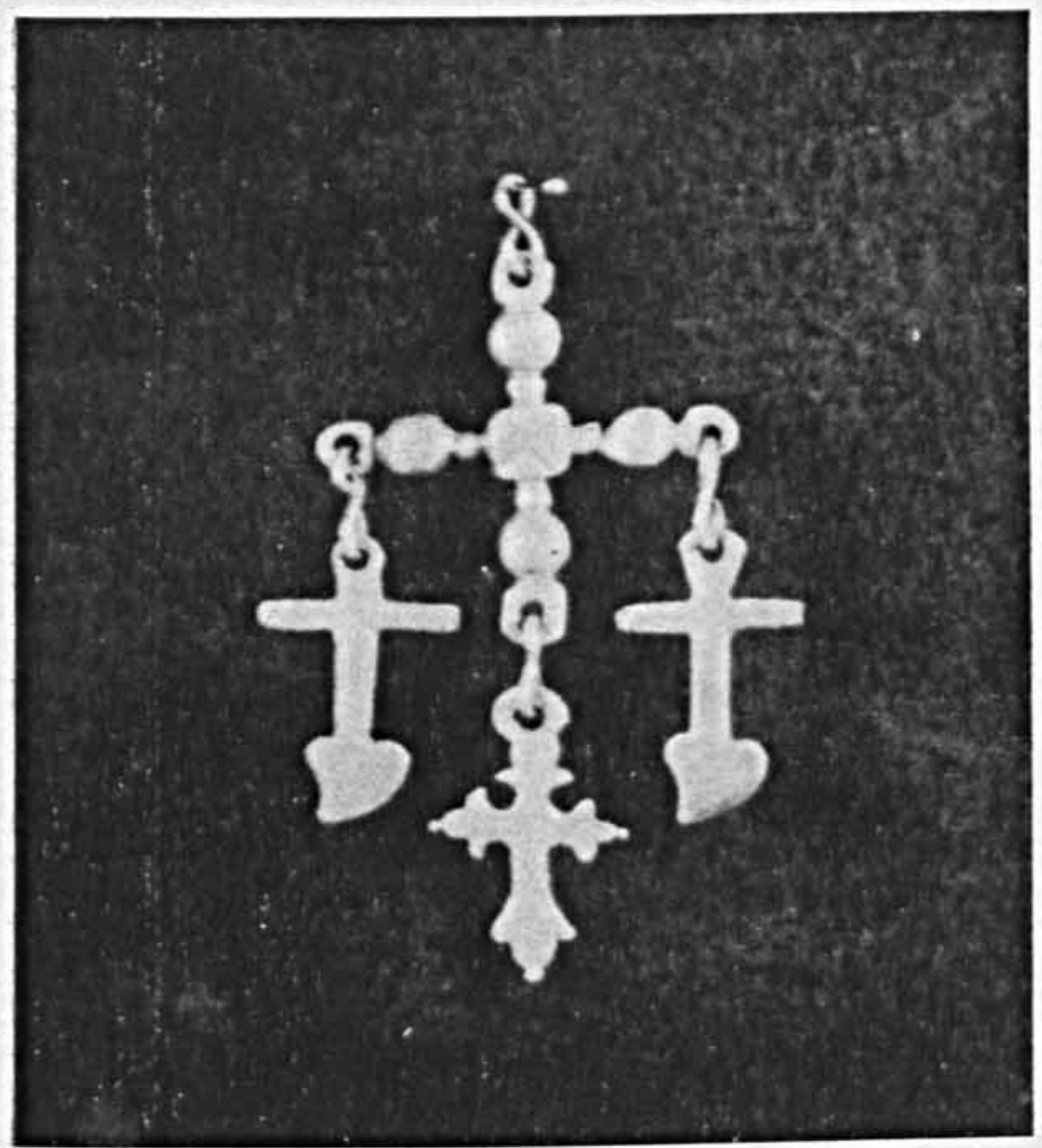

5. La cruz más pequeña de tipo moldeado. Es la misma de la fig. 2, parte inferior derecha 


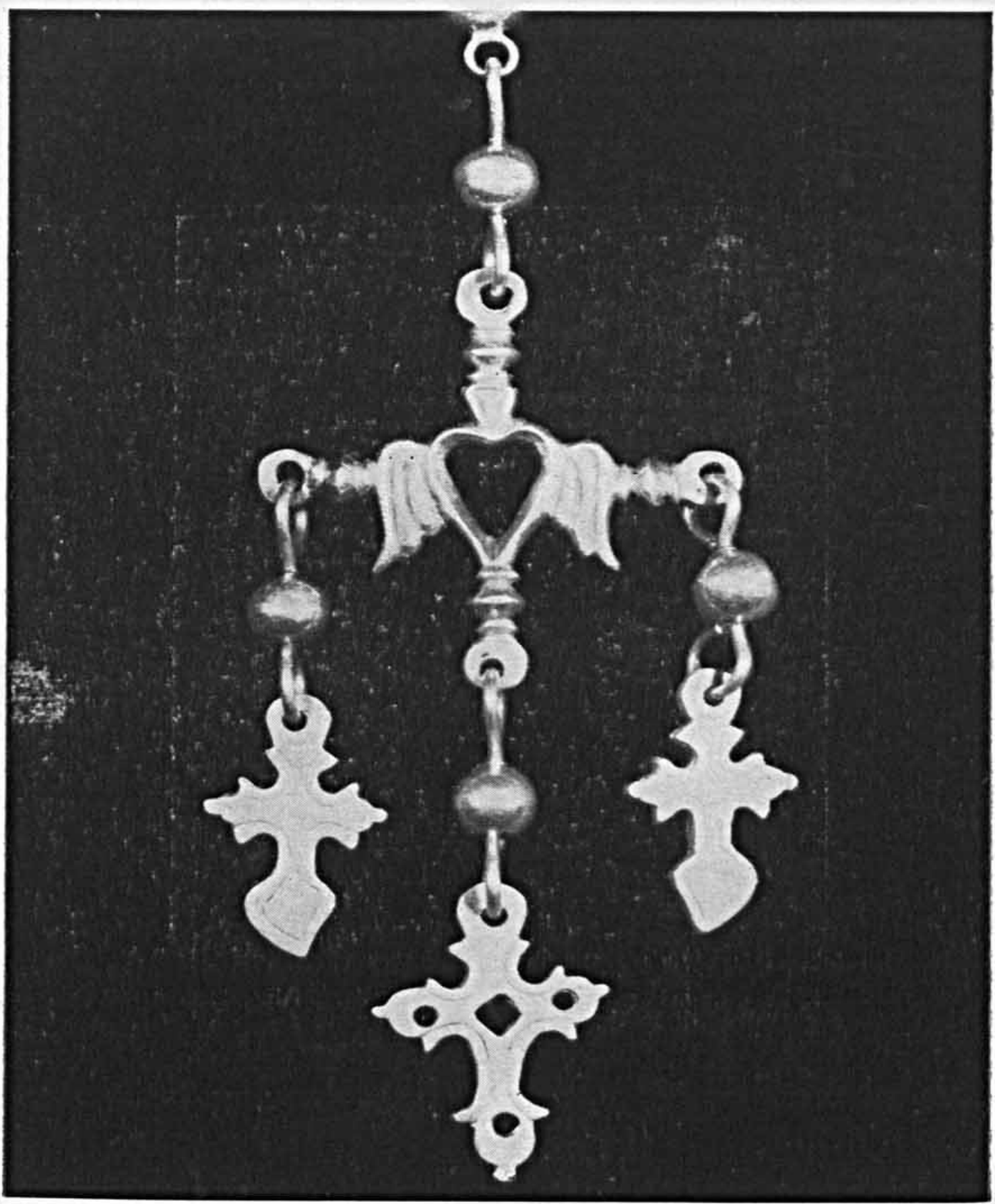

6. Cruz con el corazón de alas. (Cortesia de la señora Margarita de Rojas Garcidueñas.) 